\title{
The Effect of a Condylar Repositioning Plate on Condylar Position and Relapse in Two-Jaw Surgery
}

\author{
Gyu Sik Jung ${ }^{1}$, Taek Kyun Kim², Jeong Woo Lee ${ }^{1}$, Jung Dug Yang ${ }^{1}$, Ho Yun Chung ${ }^{1}$, \\ Byung Chae $\mathrm{Cho}^{1}$, Kang Young Choi ${ }^{1}$ \\ ${ }^{1}$ Department of Plastic and Reconstructive Surgery, Kyungpook National University School of Medicine, Daegu; ${ }^{2}$ View Plastic Surgery Clinic, \\ Seoul, Korea
}

Background Numerous condylar repositioning methods have been reported. However, most of them are 2-dimensional or are complex procedures that require a longer operation time and a highly trained surgeon. This study aims to introduce a new technique using a condylar repositioning plate and a centric relation splint to achieve a centric relationship.

Methods We evaluated 387 patients who had undergone surgery for skeletal jaw deformities. During the operation, a centric relation splint, intermediate splint, final centric occlusion splint, and condylar repositioning plate along with an L-type mini-plate for LeFort I osteotomy or a bicortical screw for bilateral sagittal split ramus osteotomy were utilized for rigid fixation. The evaluation included: a physical examination to detect preoperative and postoperative temporomandibular joint dysfunction, 3-dimensional computed tomography and oblique transcranial temporomandibular joint radiography to measure 3-dimensional condylar head movement, and posteroanterior and lateral cephalometric radiography to measure the preoperative and postoperative movement of the bony segment and relapse rate.

Results A $0.3 \%$ relapse rate was observed in the coronal plane, and a $2.8 \%$ relapse rate in the sagittal plane, which is indistinguishable from the dental relapse rate in orthodontic treatment. The condylar repositioning plate could not fully prevent movement of the condylar head, but the relapse rate was minimal, implying that the movement of the condylar head was within tolerable limits.

Conclusions Our condylar repositioning method using a centric relation splint and miniplate in orthognathic surgery was found to be simple and effective for patients suffering from skeletal jaw deformities.

Keywords Sagittal split ramus osteotomy / Le Fort osteotomy / Orthognathic surgery
Correspondence: Kang Young Choi Department of Plastic and Reconstructive Surgery, Kyungpook National University School of

Medicine, 130 Dongdeok-ro, Jung-gu, Daegu 41944, Korea

Tel: $+82-53-200-5685$

Fax: +82-53-425-3879

E-mail: kychoi@knu.ac.kr

Received: 1 Jul $2016 \bullet$ Revised: 11 Sep $2016 \bullet$ Accepted: 21 Oct 2016

pISSN: 2234-6163 • elSSN: 2234-6171 • https://doi.org/10.5999/aps.2017.44.1.19 • Arch Plast Surg 2017;44:19-25

No potential conflict of interest relevant to this article was reported.

\section{INTRODUCTION}

The goal of orthognathic surgery is to correct aesthetic and functional problems of the jaw by reshaping the maxilla and mandible within a short period. Sagittal split ramus osteotomy of the mandible is the most frequently used method for correcting retrognathic and prognathic mandibles. Rigid fixation with plates or screws has been widely favored for stabilization in the sagittal split ramus osteotomy [1]. However, this has several sequelae, including temporomandibular joint (TMJ) dysfunction 
and postoperative relapse, as the proximal condylar segment is movable in 3 dimensions after osteotomy of the mandibular ramus, which affects the condylar head position.

Many studies have investigated the factors that impact postoperative sequelae, including a displaced condylar head, method and duration of intermaxillary fixation, magnitude and direction of mandible movement, orthodontic treatment, incomplete bony union, and masticatory function [2]. It is especially evident that a displaced condylar head is a major factor. Therefore, it is important to maintain the anatomic position of the mandibular condyle within the glenoid fossa during orthognathic surgery in order to reduce the relapse rate and minimize the risk of TMJ dysfunction [3].

Numerous condylar repositioning methods have been reported, including a manual method, rigid retention, navigation, and sonographic monitoring in order to limit the movement of the condyle [4]. In addition, various means of evaluating the efficacy of these methods have been proposed, which have been largely unsuccessful due to image distortion resulting from the complex anatomic structure around the TMJ, irreproducible preoperative and postoperative images, and multidirectional displacement of the condylar head [5]. Most condylar repositioning methods are 2-dimensional or require complex procedures involving a long operation time and a highly trained surgeon.

This study aimed to introduce a new technique using a centric relation (CR) splint to achieve a centric relationship and a simple 3-dimensional condylar repositioning plate, validated by an objective evaluation method.

\section{METHODS}

The subjects were recruited from among patients who underwent surgery for skeletal jaw deformities between January 2008 and December 2011. All of them were operated on by the same surgeon. A total of 387 patients (199 male and 188 female) were followed up in our outpatient clinic for more than 1 year. The average age of the patients was 22.3 years (range, 17-52 years). The preoperative procedures included presurgical orthodontics, final surgical treatment objectives, manufacture of CR splints, and model surgery, including facebow transfer, impression model mounting, and manufacture of final intermediate and centric occlusion splints.

The sequence of operative procedures was based on the preoperative planning and model surgery. The main procedures performed were Le Fort I osteotomy and bilateral sagittal split ramus osteotomy. First, a condylar repositioning plate was applied between the maxilla and mandible after a CR splint was placed (Fig. 1A). The drilling holes for the plate were set above the Le Fort I osteotomy line and behind the sagittal split ramus osteotomy vertical line. Two 4-mm screws were usually utilized on each side to hold them firmly (Fig. 1B, C). After removing the condylar repositioning plate and CR splint, Le Fort I osteotomy was usually performed first. An intermediate splint was then used to guide the movement of one jaw relative to the other jaw. Four 4-hole L-type mini-plates were used for the Le Fort I osteotomy. The remaining jaw was then repositioned based on the final splint. Once the proximal segment of the mandible was repositioned, the condylar repositioning plate was applied on the same drilling hole and bicortical screws $2 \mathrm{~mm}$ in diameter and 12-18 mm long for bilateral sagittal split ramus osteotomy were used for rigid fixation. The condylar repositioning plate was removed immediately after the rigid fixation of the mandible [6].

The following evaluation methods were used: (1) physical examination to detect preoperative and postoperative TMJ dysfunction, (2) 3-dimensional computed tomography (3D-CT) and oblique transcranial TMJ radiography to measure 3-dimensional condylar head movement (Figs. 2, 3), and (3) standard posteroanterior and lateral cephalometric radiography to identi-

\section{Fig. 1. Operative procedure using condylar repositioning plate}

(A) Three-dimensional condylar repositioning plate. (B) Intraoperative view of the condyle repositioning plate. (C) Condyle repositioning plate on a skull model.
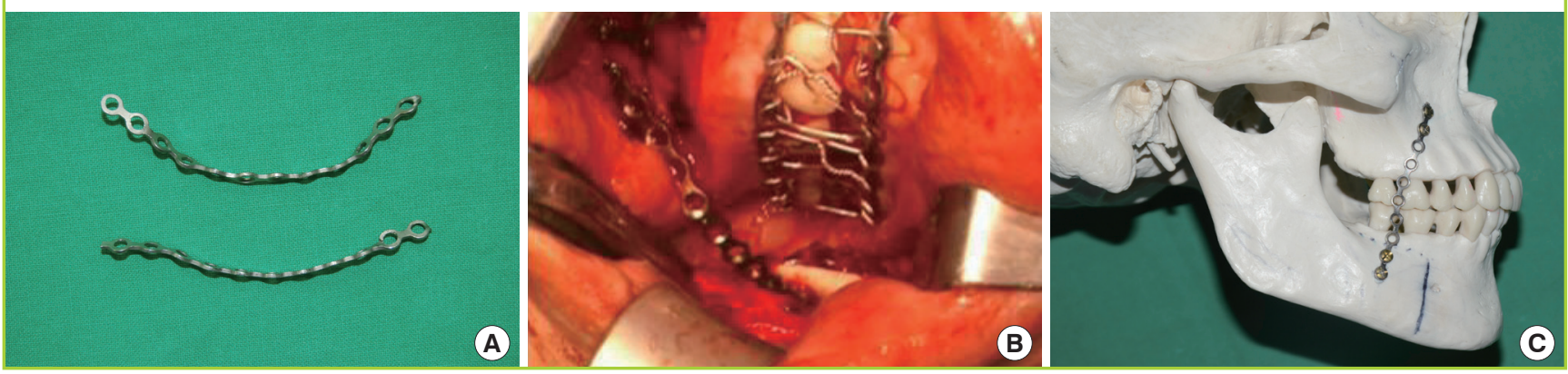


\section{Fig. 2. Three-dimensional computed tomography}

(A) Frontal view. (B) Lateral view. (C) Basal view. (D) Two-dimensional computed tomography, transverse view. FZ, line between the frontozygomatic sutures; FRA, angle between FZ and RVR; FLA, angle between FZ and LVR; RVR, right vertical ramus line; LVR, left vertical ramus line; HL, line between the external acoustic meatus and inferior orbital rim; LRA, angle between $H L$ and PR; PR, posterior ramus line; RCA, right condylar axis angle to $\mathrm{MM}^{\prime} ; \mathrm{LCA}$, left condylar axis angle to $\mathrm{MM}^{\prime} ; \mathrm{MM}^{\prime}$, line between the anterior surfaces of the mastoid process.
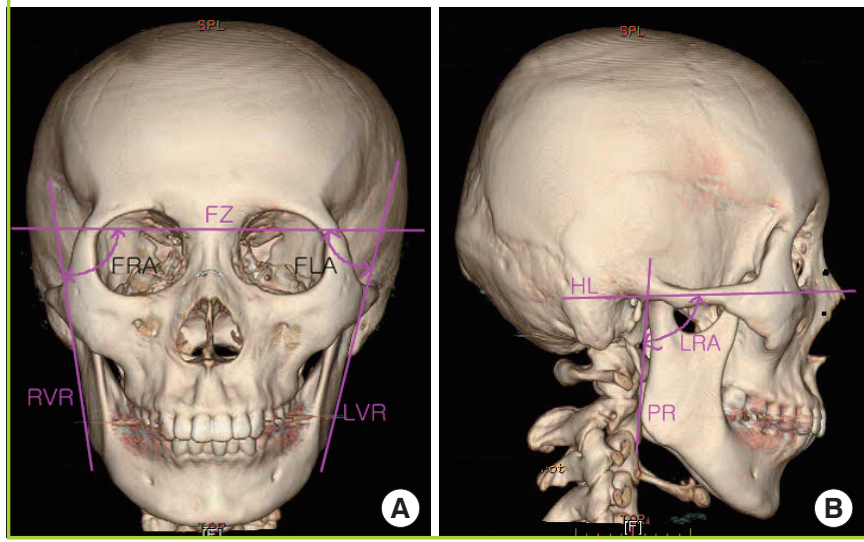

Fig. 3. Oblique transcranial temporomandibular joint radiography

FS, parietotemporal fissure; EM, anterior eminence; $X_{1}$ superior point parallel to the FS-EM line; FC1 distance, posterior joint space; FC2 distance, superior joint space; FC3 distance, anterior joint space.

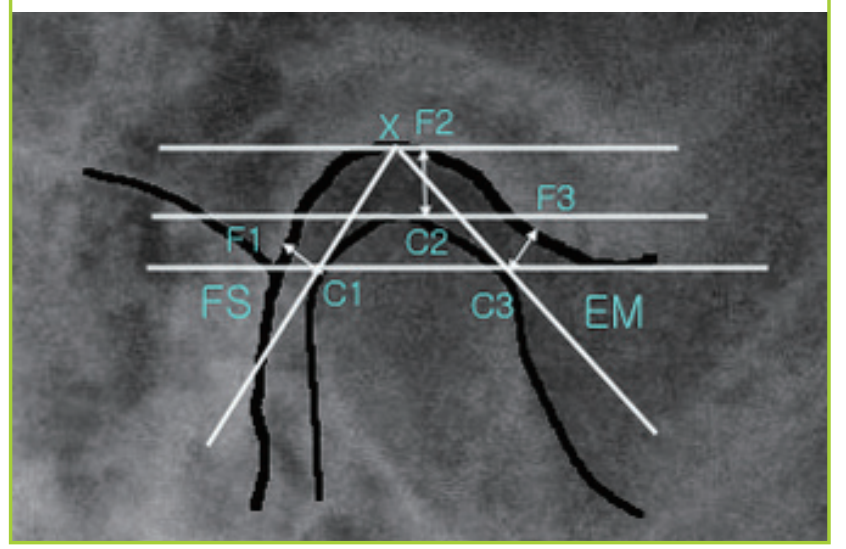

fy the amount of preoperative and postoperative movement of bony segments and the relapse rate.

The postoperative 3D-CT and other radiographs were typically taken 3 months and 1 year after the operation during followup examinations at the outpatient clinic. The radiologic findings were measured by two plastic surgeons and one orthodontist, and the mean values were used for the statistical analysis.

On the frontal view of the 3D-CT images, the angles between the line between the frontozygomatic sutures (FZ) and the vertical ramus line (VR) from both sides were measured. On the lateral view of the 3-dimensional scan, the angles between the line between the external acoustic meatus and inferior orbital $\operatorname{rim}(\mathrm{HL})$ and posterior ramus line $(\mathrm{PR})$ were measured. On
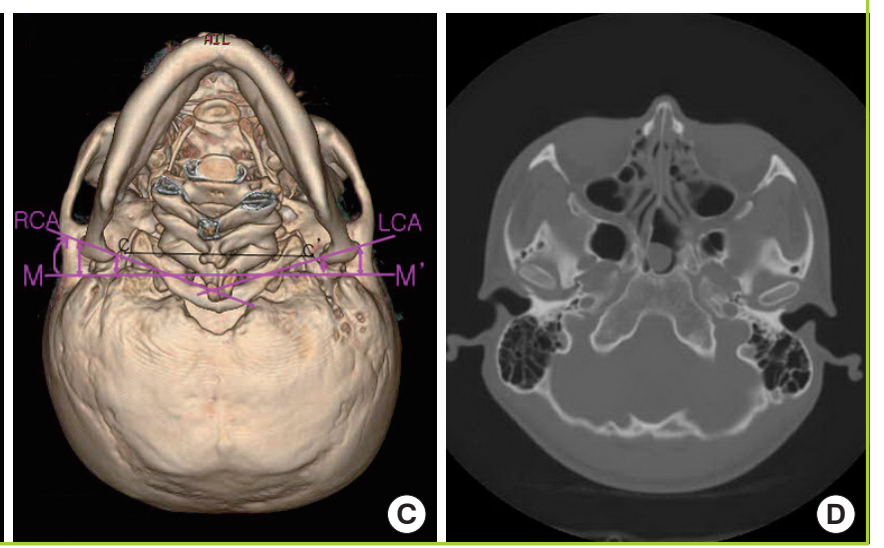

the basal view of the $3 \mathrm{D}-\mathrm{CT}$, the angles between the line between the anterior surfaces of mastoid process (MM') and the line connecting the medial and lateral ends of the condylar head were measured, along with the distances between the MM' and the outer/inner pole of the condyle on the 2-dimensional transverse view.

The landmarks were defined and abbreviated as follows: RVR, right vertical ramus line; LVR, left vertical ramus line; FRA, the angle between FZ and RVR; FLA, the angle between FZ and LVR; LRA, the angle between HL and PR; CC', the distance between the inner condylar poles; RCA, the right condylar axis angle to MM'; LCA, the left condylar axis angle to MM'; RLD, the distance between MM' to the outer pole of the right condyle; $\mathrm{RMD}$, the distance between MM' to the inner pole of the right condyle; $\mathrm{LMD}$, the distance between $\mathrm{MM}$ ' to the inner pole of the left condyle; LLD, the distance between MM' to the outer pole of the left condyle; FS, the parietotemporal fissure; $\mathrm{EM}$, the anterior eminence; and $\mathrm{X}$, the superior point parallel to the FS-SE line. Additionally, the posterior joint space (PJS) was defined as the FC1 distance, the superior joint space (SJS) as the FC2 distance, and the anterior joint space (AJS) as the FC3 distance.

Skeletal relapse was measured by changes in the position of the supramentale (B), pogonion (Pog), and menton ( $\mathrm{Mn}$ ) points, while dental relapse was measured by the changes in the position of the tip of the upper and lower incisors.

Statistical analysis was performed with SPSS Ver. 22.0. (IBM Corp. Armonk, NY, USA). A subgroup analysis was performed using the paired t-test. Statistical significance was set at $\mathrm{P}<0.01$. 


\section{RESULTS}

The average period of postoperative intermaxillary fixation was only 1 day, with no additional splint for occlusal stability. The average follow-up duration was 15.3 months. Of the $387 \mathrm{pa-}$ tients, 34 had a reciprocal click sound accompanied by TMJ pain, 78 had a closing click sound without any subjective symptoms, and the rest had no symptoms before surgery. The patients with preoperative click sounds retained them after surgery, while 6 patients exhibited a new click sound without any clinical symptoms.

In the preoperative 3D-CT scans, the average vertical axis angle was $82.73^{\circ} \pm 4.47^{\circ}$ (FRA) on the right and $83.32^{\circ} \pm 4.24^{\circ}$ (FLA) on the left on the frontal view, $84.35^{\circ} \pm 5.49^{\circ}$ (LRA) on the right and $85.58^{\circ} \pm 5.04^{\circ}$ (LLA) on the left on the lateral view, and the average long-axis angle of the condylar head was $20.93^{\circ} \pm 5.55^{\circ}(\mathrm{RCA})$ on the right and $19.89^{\circ} \pm 5.40^{\circ}$ (LCA) on the left on the basal view. In the preoperative 2-dimensional computed tomography images, the average vertical distances between the MM' and the inner and outer pole of the right condyle (RMD and RLD, respectively) were $13.48 \pm 3.86 \mathrm{~mm}$ and $19.69 \pm 4.62 \mathrm{~mm}$, respectively. The average vertical distances between the MM' to the inner and outer pole of the left condyle (LMD and LLD, respectively) were $13.31 \pm 3.34 \mathrm{~mm}$ and $19.58 \pm 3.86 \mathrm{~mm}$, respectively.

In the postoperative 3D-CT scans, the average vertical axis angle was $80.25^{\circ} \pm 4.74^{\circ}$ (FRA) on the right and $80.58^{\circ} \pm 4.30^{\circ}$ (FLA) on the left on the frontal view, $81.26^{\circ} \pm 5.72^{\circ}$ (LRA) on the right and $81.87^{\circ} \pm 5.48^{\circ}$ (LLA) on the left on the lateral view, and the average long axis angle of the condylar head was $21.79^{\circ} \pm 6.04^{\circ}$ (RCA) on the right and $21.21^{\circ} \pm 5.52^{\circ}$ (LCA) on the left on the basal view. In the postoperative $2 \mathrm{D}$-CT images, the average vertical distances between the MM' and the inner and outer pole of the right condyle (RMD and RLD, respectively) were $14.03 \pm 3.78 \mathrm{~mm}$ and $20.94 \pm 4.43 \mathrm{~mm}$, respectively. The average vertical distances between the MM' and the inner and outer pole of the left condyle (LMD and LLD, respectively) were $14.40 \pm 3.24 \mathrm{~mm}$ and $20.86 \pm 3.82 \mathrm{~mm}$, respectively.

In the long-term postoperative 3D-CT scans, the average vertical axis angle was $81.35^{\circ} \pm 4.55^{\circ}$ (FRA) on the right and $82.49^{\circ} \pm 4.21^{\circ}$ (FLA) on the left on the frontal view, $83.24^{\circ} \pm$ $5.31^{\circ}$ (LRA) on the right and $84.36^{\circ} \pm 5.53^{\circ}$ (LLA) on the left on the lateral view, and the average long axis angle of the condylar head was $21.24^{\circ} \pm 5.58^{\circ}$ (RCA) on the right and $20.72^{\circ} \pm$ $5.44^{\circ}$ (LCA) on the left on the basal view. In the long-term postoperative $2 \mathrm{D}-\mathrm{CT}$ images, the average vertical distances between the MM' and the inner and outer pole of the right condyle (RMD and RLD, respectively) were $13.56 \pm 3.83 \mathrm{~mm}$ and $20.40 \pm 4.92 \mathrm{~mm}$, respectively. The average vertical distances between the MM' and the inner and outer pole of the left condyle (LMD and LLD, respectively) were $13.84 \pm 3.18 \mathrm{~mm}$ and $20.18 \pm 3.79 \mathrm{~mm}$, respectively. Significant differences were found between the preoperative and postoperative measurements (Table 1).

In addition, the average distance at the AJS was $2.48 \pm 0.41$ $\mathrm{mm}$ on the right and $2.32 \pm 0.34 \mathrm{~mm}$ on the left, the average distance at the SJS was $2.32 \pm 0.39 \mathrm{~mm}$ on the right and $2.40 \pm 0.36$ $\mathrm{mm}$ on the left, and the average distance at the PJS was

Table 1. Mean measurements and statistical results of preoperative, 3-month postoperative, and 1-year postoperative 3-dimensional and 2-dimensional computed tomography imaging

\begin{tabular}{|c|c|c|c|c|c|c|}
\hline \multirow{2}{*}{ Measurement } & \multirow{2}{*}{ T0 (mean) } & \multirow{2}{*}{ T1 (mean) } & \multirow{2}{*}{ TL (mean) } & \multicolumn{3}{|c|}{ Paired t-test } \\
\hline & & & & T0-T1 & $\mathrm{T} 1-\mathrm{TL}$ & T0-TL \\
\hline FRA & 82.73 & 80.25 & 81.35 & $<0.01^{*}$ & $<0.01^{*}$ & $<0.01^{*}$ \\
\hline FLA & 83.32 & 80.58 & 82.49 & $<0.01^{*}$ & $<0.01^{*}$ & $<0.01^{*}$ \\
\hline LRA & 84.35 & 81.26 & 83.24 & $<0.01^{*}$ & $<0.01^{*}$ & $<0.01^{*}$ \\
\hline LLA & 85.58 & 81.87 & 84.36 & $<0.01^{*}$ & $<0.01^{*}$ & $<0.01^{*}$ \\
\hline RCA & 20.93 & 21.79 & 21.24 & $<0.01^{*}$ & $<0.01^{*}$ & 0.03 \\
\hline LCA & 19.89 & 21.21 & 20.72 & $<0.01^{*}$ & 0.03 & $<0.01^{*}$ \\
\hline RLD & 19.69 & 20.94 & 20.40 & $<0.01^{*}$ & 0.12 & 0.02 \\
\hline $\mathrm{RMD}$ & 13.48 & 14.03 & 13.56 & $<0.01^{*}$ & $<0.01^{*}$ & 0.18 \\
\hline LMD & 13.31 & 14.40 & 13.84 & $<0.01^{*}$ & $<0.01^{*}$ & $<0.01^{*}$ \\
\hline LLD & 19.58 & 20.86 & 20.18 & $<0.01^{*}$ & $<0.01^{*}$ & $<0.01^{*}$ \\
\hline
\end{tabular}

T0, preoperative; T1, 3 months postoperative; TL, 1 year postoperative; FRA, angle between FZ and RVR; FLA, angle between FZ and LVR; LRA, angle between HL and PR on the right on the lateral view; LLA, angle between HL and PR on the left on the lateral view; RCA, right condylar axis angle to MM'; LCA, left condylar axis angle to MM'; RLD, distance between MM' and the outer pole of the right condyle; RMD, distance between MM' and the inner pole of the right condyle; LMD, distance between MM' and the inner pole of the left condyle; LLD, distance between MM' and the outer pole of the left condyle; FZ, line between the frontozygomatic sutures; RVR, right vertical ramus line; LVR, left vertical ramus line; HL, line between the external acoustic meatus and inferior orbital rim; PR, posterior ramus line; MM', line between the anterior surfaces of the mastoid process.

${ }^{*} \mathrm{P}<0.01$. 
$2.35 \pm 0.37 \mathrm{~mm}$ on the right and $2.43 \pm 0.41 \mathrm{~mm}$ on the left in the preoperative oblique transcranial TMJ radiographs. In the postoperative oblique transcranial TMJ radiography, the average distances at the AJS were $2.25 \pm 0.41 \mathrm{~mm}$ on the right and $2.12 \pm 0.38 \mathrm{~mm}$ on the left, $2.66 \pm 0.37 \mathrm{~mm}$ on the right and $2.68 \pm 0.43 \mathrm{~mm}$ on the left at the SJS, and $2.73 \pm 0.41 \mathrm{~mm}$ on the right and $2.76 \pm 0.40 \mathrm{~mm}$ on the left at the PJS. In the longterm postoperative oblique transcranial TMJ radiography, the average distance was $2.43 \pm 0.38 \mathrm{~mm}$ on the right and $2.29 \pm$ $0.36 \mathrm{~mm}$ on the left at the AJS, $2.46 \pm 0.34 \mathrm{~mm}$ on the right and $2.50 \pm 0.37 \mathrm{~mm}$ on the left at the SJS, and $2.46 \pm 0.37 \mathrm{~mm}$ on the right and $2.52 \pm 0.36 \mathrm{~mm}$ on the left at the PJS. Statistically significant differences were found between the preoperative and postoperative measurements (Table 2).

Standard anteroposterior and lateral cephalic radiography scans were performed in order to analyze 3-dimensional bony movement and relapse. The reference points were the sella turcica (S), nasion (N), B, Pog, Mn, and the vertical reference plane (VP). The VP was orthogonal to the horizontal reference plane (HP), which was rotated $7^{\circ}$ clockwise from the line connecting $S$ and N. The distances from VP to Po and Mn were designated as $\mathrm{hPo}$ and $\mathrm{hMn}$, respectively. The distances from $\mathrm{N}$ to the projections of Po and $\mathrm{Mn}$ onto the VP were designated as vPo and vMn, respectively.

In the preoperative images, the mean $\mathrm{SNB}$ angle was $81.34^{\circ} \pm$ $6.24^{\circ}$, hPo was $-2.95 \pm 14.62 \mathrm{~mm}$, hMn was $-10.34 \pm 14.83 \mathrm{~mm}$, vPo was $126.72 \pm 8.66 \mathrm{~mm}$, and vMn was $134.31 \pm 8.95 \mathrm{~mm}$. In the postoperative images, the mean angle was $78.04^{\circ} \pm 4.85^{\circ}, \mathrm{hPo}$ was $-8.45 \pm 10.91 \mathrm{~mm}$, hMn was $-14.98 \pm 11.27 \mathrm{~mm}$, vPo was $125.97 \pm 7.89 \mathrm{~mm}$, and vMn was $132.74 \pm 7.94 \mathrm{~mm}$. In the longterm postoperative images, the mean angle was $78.31^{\circ} \pm 4.85^{\circ}$, hPo was $-8.22 \pm 10.77 \mathrm{~mm}$, hMn was $-14.58 \pm 11.74 \mathrm{~mm}$, vPo was $125.54 \pm 7.56 \mathrm{~mm}$, and vMn was $132.38 \pm 7.76 \mathrm{~mm}$.

A relapse rate of $0.3 \%$ was observed in the coronal plane, while a relapse rate of $2.8 \%$ was observed in the sagittal plane. This cannot be distinguished from the dental relapse rate in orthodontic treatment (Table 3). The condylar repositioning plate was unable to fully prevent the movement of the condylar head, but the relapse rate was minimized. This implies that the movement of the condylar head was controlled within tolerable limits.

\section{DISCUSSION}

Many clinicians are concerned that rigid internal fixation can in-

Table 2. Mean distance of joint space on oblique transcranial radiographs

\begin{tabular}{|lcccccc|}
\hline \multirow{2}{*}{ Measurement, $\mathbf{m m}$} & T0 (mean) & T1 (mean) & TL (mean) & \multicolumn{3}{c|}{ Paired t-test } \\
\cline { 5 - 7 } & & & & \multicolumn{2}{c}{ T0-T1 } & T1-TL \\
\hline AJS (R) & 2.48 & 2.25 & 2.43 & $<0.01^{*}$ & $<0.01^{*}$ & 0.02 \\
AJS (L) & 2.32 & 2.12 & 2.29 & $<0.01^{*}$ & $<0.01^{*}$ & 0.34 \\
SJS (R) & 2.32 & 2.66 & 2.46 & $<0.01^{*}$ & $<0.01^{*}$ & $<0.01^{*}$ \\
SJS (L) & 2.40 & 2.68 & 2.50 & $<0.01^{*}$ & $<0.01^{*}$ & $<0.01^{*}$ \\
PJS (R) & 2.35 & 2.73 & 2.46 & $<0.01^{*}$ & $<0.01^{*}$ & $<0.01^{*}$ \\
PJS (L) & 2.43 & 2.76 & 2.52 & $<0.01^{*}$ & $<0.01^{*}$ & $<0.01^{*}$ \\
\hline
\end{tabular}

T0, preoperative; T1, 3 months postoperative; TL, 1 year postoperative; AJS, anterior joint space; (R), right side; (L), left side; SJS, superior joint space; PJS, posterior joint space.

${ }^{*} \mathrm{P}<0.01$.

Table 3. Changes in landmarks on lateral cephalic radiographs

\begin{tabular}{|c|c|c|c|c|c|c|}
\hline \multirow{2}{*}{ Measurement, $\mathrm{mm}$} & \multirow{2}{*}{ T0 (mean) } & \multirow{2}{*}{ T1 (mean) } & \multirow{2}{*}{ TL (mean) } & \multicolumn{3}{|c|}{ Paired t-test } \\
\hline & & & & T0-T1 & T1-TL & T0-TL \\
\hline SNB & 81.34 & 78.04 & 78.31 & $<0.01^{*}$ & 0.05 & $<0.01^{*}$ \\
\hline $\mathrm{hPo}$ & -2.95 & -8.45 & -8.22 & $<0.01^{\star}$ & 0.45 & $<0.01^{*}$ \\
\hline $\mathrm{hMn}$ & -10.34 & -14.98 & -14.58 & $<0.01^{*}$ & 0.50 & $<0.01^{*}$ \\
\hline vPo & 126.72 & 125.97 & 125.54 & 0.21 & 0.08 & 0.04 \\
\hline $\mathrm{VMn}$ & 134.31 & 132.74 & 132.38 & 0.01 & 0.05 & $<0.01^{*}$ \\
\hline \multicolumn{7}{|c|}{$\begin{array}{l}\text { The reference points were the sella turcica }(\mathrm{S}) \text {, nasion }(\mathrm{N}) \text {, supramentale }(\mathrm{B}) \text {, pogonion }(\mathrm{Pog}) \text {, menton }(\mathrm{Mn}) \text {, and the vertical reference plane (VP). The VP was orthogonal to } \\
\text { the horizontal reference plane (HP), which was rotated } 7^{\circ} \text { clockwise from the line connecting } \mathrm{S} \text { and } \mathrm{N} \text {. The distances from VP to Po and Mn were designated as hPo and } \\
\text { hMn, respectively. The distances from N to the projections of Po and Mn onto VP were designated as vPo and vMn, respectively. } \\
\text { T0, preoperative; T1, } 3 \text { months postoperative; TL, } 1 \text { year postoperative. } \\
\text { *P }<0.01 \text {. }\end{array}$} \\
\hline
\end{tabular}


duce significant changes in the position of the condyle. Although the use of condylar positioning devices (CPDs) seems reasonable from this point of view, no critical evaluation of their use is currently available. In particular, the effects of a condylar repositioning plate on condylar position and relapse have never been studied in detail. In a review by Costa et al. of the English-language literature since 1990, only 6 papers comparing the use of CPDs with traditional methods were found since a comprehensive review on the use of CPDs in orthognathic surgery was published in 1994 by Ellis $[4,7]$.

To summarize these 6 studies, the outcomes of 141 patients with CPDs were compared with those of 112 patients treated using conventional manual repositioning. Three studies supported the use of CPDs, 1 study supported the use of CPDs only in patients with temporomandibular disorders (TMDs), and 2 studies did not support the use of CPDs because they failed to improve skeletal stability or TMJ function, irrespective of the skeletal deformities treated [4,8-13].

In our study, for the determination of acceptable patient outcomes and patient suitability for the procedure, we used broader criteria than those used in previous studies of orthognathic surgery that used only simple numeric criteria. The criteria took into account the patient's aesthetic requirements as the most important factor, followed in order by morphological esthetics, jaw deformities not being overcome by correction, and asymmetry exceeding $3 \mathrm{~mm}$ and $3^{\circ}$. In order to statistically verify the outcomes with multiple variables, a large sample size and a sufficient follow-up period were incorporated into the study design.

With respect to outcome evaluation, X-ray based methods have been used frequently, and CT-based methods are also used. Accordingly, we used both X-ray and CT imaging.

The present study was not designed as a comparative study between a group that used CRP and a group that did not use CRP. Instead, it was a study of the usefulness of a novel method of CRP design and placement, in contrast to the overly complicated conventional methods currently in use. Now that we have shown that this novel method was successful and not time-consuming, a future study is being planned to compare this group to those who did not use a CRP.

The time required in using a CRP was not measured each time, but it generally took 5-10 minutes for the first CRP and 1-2 minutes to fix the distal segment to the proximal segment and to fix the sagittal split ramus osteotomy. This occurs because the proximal segment is fixed to the upper jaw, meaning that it is more stable than fixation without a CRP, which consequently shortens the time for fixation to the lower jaw. Therefore, it is believed that this novel method has virtually no effect on operative time. However, it should be mentioned that the
CR splint must be produced prior to the surgery, which requires approximately 20-30 minutes.

The key goal of this article was to establish the usefulness of using a CRP. Although various factors are involved, we analyzed a large number of patients treated by a single surgeon when studying the effects of the CRP, in order to link measureable outcomes to the effects of the CRP. Of course, this study has limitations, but to study all the factors that could affect this operation would be very difficult. Accordingly, the present study aimed to clarify the effects of CRP as meaningfully as possible.

Controversy exists about the appropriate management of patients with preexisting TMDs who require orthognathic surgery for the correction of malocclusion and jaw deformities. Two significantly different philosophies exist: although Wolford et al. [14] contended that orthognathic surgical procedures help in the reduction of TMD dysfunction and symptoms, Cottrell et al. [15] showed that orthognathic surgery in such patients caused further deleterious effects on the TMD and thus worsened the symptoms and dysfunction after surgery. The latter philosophy proposes surgical management of the TMD pathology as an initial separate procedure or one that may be performed concomitantly with orthognathic surgery when indicated [14]. However, a retrospective analysis has suggested that orthognathic surgery itself improves the symptoms of TMD. In that analysis, 53\% of patients undergoing orthognathic surgery had signs and/or symptoms of TMD and 78\% of those patients reported an improvement in symptoms after surgery [16]. These findings are, however, not applicable to this study because the patients had no clinical problems and were accustomed to a symptom-free TMJ.

In this study, the effect of the condylar repositioning plate on condylar position and relapse was evaluated by the paired t-test and regression. The condylar repositioning plate could not entirely prevent the movement of the condylar head, but did minimize the relapse rate according to our statistical analysis. This implies that the condylar head movement was within tolerable limits.

Although some researchers do not support the use of CPDs, their main objection is not the precision of such devices, but rather the amount of time that they add to procedures. Once less time-consuming methods are available, there are likely to be few objections to the use of CPDs. Our condylar repositioning method using a CR splint and mini-plate in orthognathic surgery was simple and effective in patients suffering from skeletal jaw deformities. The CT scans, transcranial TMJ radiographs, and cephalometric radiographs all confirmed that condylar head movement was within acceptable limits. Therefore, we believe that this method is effective and reliable. 


\section{REFERENCES}

1. Harada K, Okada Y, Nagura H, et al. A new condylar positioning appliance for two-jaw osteotomies (Le Fort I and sagittal split ramus osteotomy). Plast Reconstr Surg 1996; 98:363-5.

2. Choi KY, Lee SH. Evaluation of condylar position using computed tomography following bilateral sagittal split ramus osteotomy. J Korean Assoc Maxillofac Plast Reconstr Surg 1996;18:570-93.

3. Baek RM, Yoon IM. Condylar positioning in sagittal split ramus osteotomy by the triangle method. Ann Plast Surg 2005;55:335-7.

4. Costa F, Robiony M, Toro C, et al. Condylar positioning devices for orthognathic surgery: a literature review. Oral Surg Oral Med Oral Pathol Oral Radiol Endod 2008;106: 17990.

5. Kundert M, Hadjianghelou O. Condylar displacement after sagittal splitting of the mandibular rami: a short-term radiographic study. J Maxillofac Surg 1980;8:278-87.

6. Patel PK, Novia MV. The surgical tools: the LeFort I, bilateral sagittal split osteotomy of the mandible, and the osseous genioplasty. Clin Plast Surg 2007;34:447-75.

7. Ellis E 3rd. Condylar positioning devices for orthognathic surgery: are they necessary? J Oral Maxillofac Surg 1994;52: 536-52.

8. Gerressen M, Stockbrink G, Smeets R, et al. Skeletal stability following bilateral sagittal split osteotomy (BSSO) with and without condylar positioning device. J Oral Maxillofac Surg 2007;65:1297-302.

9. Gerressen M, Zadeh MD, Stockbrink G, et al. The functional long-term results after bilateral sagittal split osteotomy (BSSO) with and without a condylar positioning device. J Oral Maxillofac Surg 2006;64:1624-30.

10. Landes CA, Sterz M. Evaluation of condylar translation by sonography versus axiography in orthognathic surgery patients. J Oral Maxillofac Surg 2003;61:1410-7.

11. Renzi G1, Becelli R, Di Paolo C, et al. Indications to the use of condylar repositioning devices in the surgical treatment of dental-skeletal class III. J Oral Maxillofac Surg 2003;61: 304-9.

12. Helm G, Stepke MT. Maintenance of the preoperative condyle position in orthognathic surgery. J Craniomaxillofac Surg 1997;25:34-8.

13. Rotskoff KS, Herbosa EG, Villa P. Maintenance of condyleproximal segment position in orthognathic surgery. J Oral Maxillofac Surg 1991;49:2-7.

14. Wolford LM, Reiche-Fischel O, Mehra P. Changes in temporomandibular joint dysfunction after orthognathic surgery. J Oral Maxillofac Surg 2003;61:655-60.

15. Cottrell DA, Edwards SP, Gotcher JE. Surgical correction of maxillofacial skeletal deformities. J Oral Maxillofac Surg 2012;70:e107-36.

16. Upton LG, Scott RF, Hayward JR. Major maxillomandibular malrelations and temporomandibular joint pain-dysfunction. J Prosthet Dent 1984;51:686-90. 